\title{
COMMENT LE CHILI DEVELOPPE-T-IL DES STRATEGIES DE COMMUNICATION VITIVINICOLE?
}

\author{
E. Rouvellac \\ Université de Limoges - Faculté des Lettres et des Sciences Humaines \\ 39E, rue Camille Guérin - 87036 Limoges cedex - France \\ UMR CNRS 6042 GEOLAB \\ eric.rouvellac@unilim.fr
}

Resumen: La viticultura en Chile se inició en la época de los conquistadores, pero su modernización y acceso a la exportación masiva es reciente, datando de los años 80. Sin un mercado nacional importante, el país construye poco a poco una comunicación efectiva para vender sus vinos en el extranjero. Es así que, por un lado, el aislamiento se utiliza como herramienta de comunicación con el pretexto sanitario de ausencia de influencias exteriores. Por ejemplo, la protección frente a la filoxera se emplea como una importante herramienta de comunicación, o se ha mantenido la singularidad alrededor de la cepa Carménère. Por otro lado, se desarrolla una comunicación alrededor del viñedo y del vino inspirada por una arquitectura iconoclasta para fortalecer el enoturismo. La comunicación también se orienta hacia el medio ambiente, con el objetivo de considerar el proceso de elaboración del vino como factor de desarrollo sostenible ¿Eso es verdad o se trata de "lavado verde"? Los resultados muestran que el medio ambiente es una importante herramienta al servicio de la comunicación.

Palabras clave: comunicación vitivinícola, cepa Carménère, arquitectura de bodega, enoturismo, desarrollo sostenible, Chile.

\begin{abstract}
The vine growing in Chile is a history rising in conquistadors' times, but the modernization of this one and its access to the massive export is recent, going back to 1980's. Without internal market worthy of the name, the country builds little by little an effective communication to sell its wines abroad. It is so on one hand that the isolation is used as communications tool with the sanitary pretext of absence of outside influences. For
\end{abstract}

Recibido: 02-12-2015. Aceptado: 28-10-2016. 
example the conservation of the phylloxera is moved forward as a strong communication tool, or the originality was maintained around the vine Carménère. On the other hand is developed a communication around the vineyard and around the wine, being inspired by the architecture iconoclastic as a vineyard and wine entrance or of the strength of the winemaking tours. The communication is also turned towards the environment, with the objective to respect a winemaking charter in sustainable development. Is it the real will or only greenwashing? The results in half complexion show especially that the environment is a tool in the service of the communication.

Keywords: wine-making communication, isolation, grafting, vine Carménére, architecture of winery, wine-making tour, sustainable development, Chile.

\section{Introduction}

Pays viticole depuis les conquistadors au XVIème siècle, le Chili n'émerge à l'échelle mondiale qu'à partir des années 1970-1980, passant de 5 à 10 millions d'hl de production de vin de 1970 à 2011, (OIV, 2015, figure 1). Ce dynamisme productif et exportateur du pays depuis presque 40 ans, (70\% du vin étaient exportés en 2012), prend sa source dans une volonté politique de s'inscrire dans une économie de libre échange, élaborée à partir du régime dictatorial incarné par le général Pinochet. Le vignoble a ainsi vu sa superficie passer de 120000 ha en 1995 à 202000 en 2013, une augmentation de presque $70 \%$ en un peu moins de 20 ans, figure 2 .

Le Chili viticole est souvent sujet aux superlatifs par ses observateurs, contrastes paysagers extraordinaires grâce à la proximité entre les sommets de la Cordillère des Andes à plus de $6000 \mathrm{~m}$ et les étroites plaines et collines bordières de l'océan Pacifique, contrastes latitudinaux entre désert et zone tempérée grâce à l'étirement du Pays, explosion de la production vitivinicole, apprentissage de la qualité en temps record, force de l'exportation.

Les superlatifs employés, (Hinnewinkel et Vélasco-Graciet, 2005; 2007, Schirmer, 2005), portent autant sur les conditions naturelles que sur l'émergence commerciale des vins chiliens sur le marché international. Le vignoble est divisé traditionnellement en vallées dont les principales productrices sont la vallée de Maïpo, entourant la capitale, où la vigne est parfois en rude concurrence avec l'urbanisme, mais où on produit toutes sortes de vins. Au nord dans le désert de l'Atacama, se trouve par exemple la vallée de l'Aconcagua où est cultivé principalement du Cabernet-Sauvignon et depuis les années 1990 la Syrah. A l'ouest de la capitale avec la vallée de Casablanca et Valparaiso apparaissent principalement des blancs à partir de Chardonnay et de Sauvignon. Dans 
la Colchagua au sud sont produit essentiellement des vins rouges à partir de CabernetSauvignon, Dans la vallée de Curico le rouge domine, et dans le Maule, qui concentre 43\% de la superficie plantée du pays, le cépage traditionnel Pais, bien qu'encore très rependu, laisse la place aux Cabernets, Merlot et Carménère.

Le Chili ne consomme qu'un tiers de sa production de vin, alors qu'il en consommait 10\% de plus il y a 15 ans. Il est condamné à exporter dans un contexte mondial toujours plus concurrentiel. La part du Nouveau Monde vitivinicole dans l'exportation du vin est passé de 10 à plus de 30\% ces 15 dernières années. Le pays a intensifié ses exportations vers ses débouchés habituels, 13\% de ses vins partent aux Etats-Unis, 11\% en Grande Bretagne, $4 \%$ au Canada, voilà les principaux pays vers qui exporte le Chili. Cette volonté d'écouler la production passe par des politiques de communications intérieures et extérieures. Dans le pays même, le développement de l'œenotourisme est un enjeu important. Il s'organise avec des tours viticoles assez efficaces mais seulement pour l'instant autour de la capitale Santiago. Ces visites de domaines ventent les atouts de l'isolement sanitaire du pays et mettent en avant des moyens mondialisés de communication comme celui autour de l'architecture particulière de certains chais. A l'extérieur, devenir le premier exportateur mondial constitue le défi à relever par les autorités chiliennes dans un horizon proche, 2020. Une communication et une charte très portées sur l'écologie et les droits humains des travailleurs de la filière sont mises en place. Quelles sont donc les stratégies de communication vitivinicole sur lesquelles s'appuie le Chili pour agrandir son marché intérieur et surtout ses exportations de vins?

Pour cela, le pays a augmenté ses capacités et son panel de communication, il allie des aspects classiques, assez déterministes, bâtis sur les spécificités environnementales d'un l'isolement relatif, à une histoire viticole liée à cet isolement supposé imperméable. Le Chili emprunte aussi à ses voisins du Nouveau monde une vision postmoderne de la communication en l'axant non pas sur le vins, la vigne ou les terroirs, mais par exemple sur l'architecture iconoclaste de certaines de ces bodegas. Le pays sait osciller entre tradition et (post)modernisme en proposant toujours une communication basée sur le classicisme colonial de ces domaines les plus anciens lors de tours viticoles. Enfin, il essaye d'utiliser la mode du développement durable à des fins de communication et de marketing en ayant l'objectif de devenir le premier pays mondial exportateur de vin dans la future décennie.

\section{L'isolement comme outil de communication}

\subsection{Le prétexte sanitaire de l'isolement des influences extérieures}

Ce sont le vide et la virginité d'influences humaines qui demeurent mis en avant pour argumenter sur un isolement préservant le vignoble chilien des risques sanitaires. Il y aurait le vide du désert de l'Atacama au nord, des Andes à l'est, de la Patagonie et de 




Figura 1. Présentation des régions viticoles chiliennes.

Source: F. Cerbelaud, E. Rouvellac, 2015.

l'Antarctique au sud, du Pacifique à l'ouest. Cette idée est renforcée par la présentation des Andes comme une barrière naturelle, du Pacifique ou de l'Antarctique comme des territoires quasi vierges. L'immensité de ces territoires vides ou presque d'hommes serait un gage d'isolement du pays qui renforcerait une sureté sanitaire, face aux menaces de l'extérieur, face aux maladies comme le phylloxéra pour la vigne, ou l'encéphalite spongiforme pour les bovins. Ces arguments autour des vertus de l'isolement du Chili, géographiques et déterministes, s'appuient sur une étymologie même du mot Chili, entre autres hypothèses, venant de l'Aymara : "là où se termine la terre "1.

1. L'Aymara est la langue des Incas, ce serait l'Inca Tupac Yupanqui (décédé en 1493) qui aurait donné ce nom aux terres conquises au sud de son empire. 

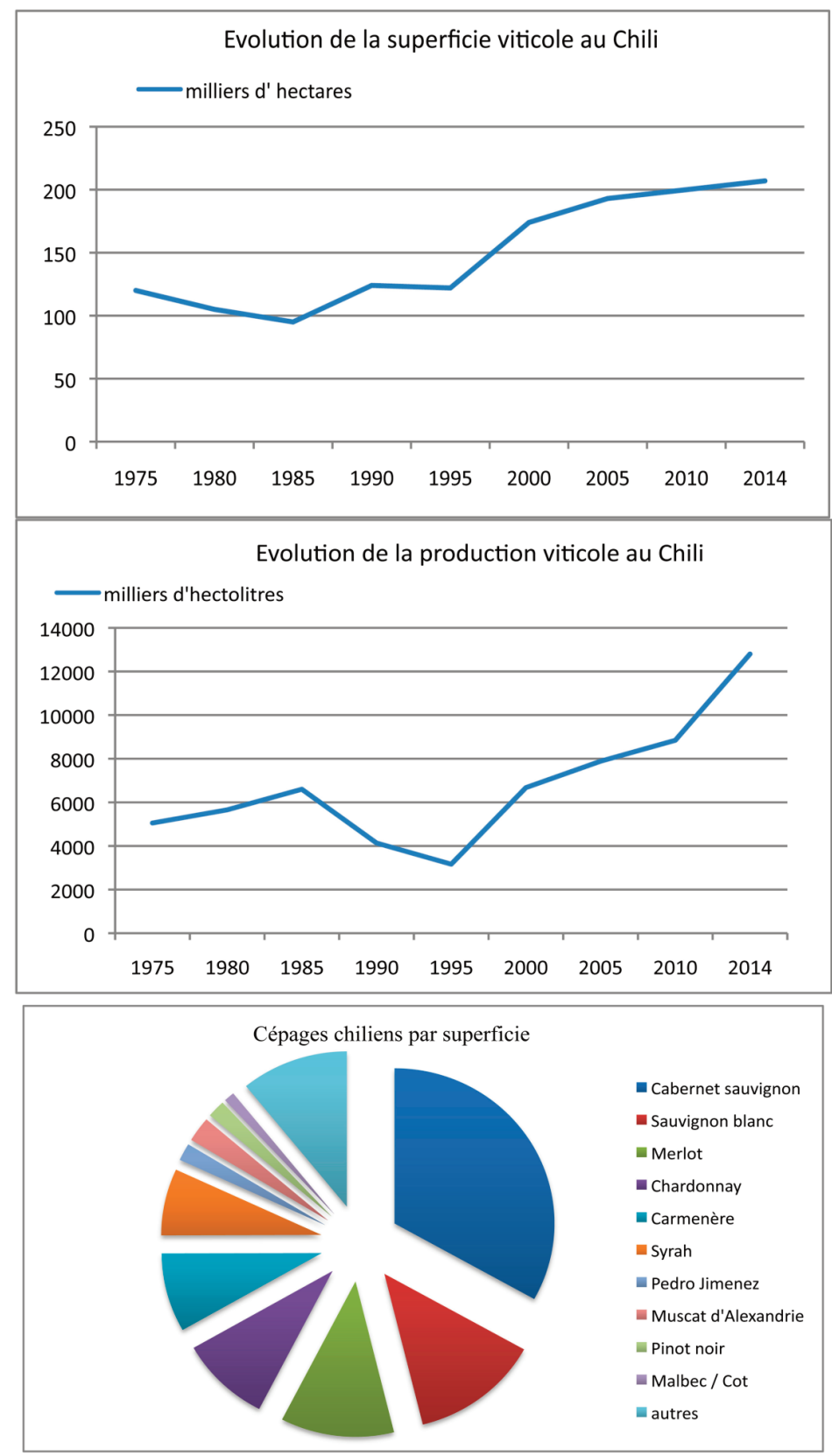

Figura 2. Evolution du vignoble chilien depuis 40 ans. Source: E. Rouvellac, 2015. 
Ce plaidoyer dénote de tout un discours qui s'appuie sur ces notions du bout du monde d'isolement, de territoire préservé, de pureté induite, notamment des maladies, pour mettre en valeur les spécificités du vignoble chilien, sain, préservé, donc unique. Mais ni le désert de l'Atacama, ni les Andes, aisément franchissable à proximité de Santiago, la capitale, ni la Patagonie ne sont vides, même si les densités humaines y restent faibles. Les échanges existent, et demeurent même intense à travers la façade portuaire qu'offre le pays.

Ce qui est récurrent au Chili, c'est aussi l'isolement politique, avec une instabilité politique durant tout le XXème siècle, culminant avec la dictature de Pinochet de 1973 à 1989. Pourtant la politique virulente d'exportation vinicole a été initiée par le régime militaire dès le début des années 1980, (Tulet et al. 2002, Paegelow et al. 2008, Salomon, 2005). A l'issue de ces années noires, le pays a connu une situation politique stable qui a attiré et attire les investisseurs. Il est devenu le premier pays au monde en matière d'ouverture de son marché avec la signature d'accords de libre échange avec quasiment toutes les régions du Monde. Sa législation favorise l'investissement étranger en le traitant à égalité avec l'investissement local. Ces dispositions économiques essentiellement libérales montrent que la communication géographique du Chili viticole n'est qu'un vernis pour favoriser l'imaginaire du consommateur.

L'isolement géographique, savamment construit dans le cadre de sa communication, cache en fait une politique de restrictions douanières avec laquelle le pays tente de se préserver de l'encéphalite spongiforme bovine, ou du phylloxéra, prétexte particulier de communication en lui-même à propos de la vitiviniculture chilienne.

\subsection{La préservation du phylloxéra comme outil de communication}

Le Chili, ou l'Argentine, peuvent s'enorgueillir de pouvoir planter la vigne franche de pied, c'est à dire de s'abstenir de greffer le cépage, le plus souvent européen, sur un porte greffe américain. C'est au prix du greffage que la vigne pousse sans subir la destruction du phylloxera, l'insecte qui a conduit à une complète destruction, un complet remodelage de la vigne et de son économie après son introduction en Europe vers 1860.

Pas de phylloxera au Chili à cause de quoi ? L'isolement est convoqué comme nous l'avons vu précédemment, bien que la maladie soit signalée au Pérou frontalier en 1888. Le peu de contact avec les autres pays serait une cause principale de l'absence de l'insecte. Comment expliquer alors que l'Australie, ou la Nouvelle Zélande, pays viticoles insulaires plus isolés que le Chili, aient connu l'épidémie dès 1875 ? Le phylloxera a même été signalé à nouveau en Nouvelle Zélande en 2006.

La nature des sols est aussi évoquée avec leur richesse en cuivre, naturelle ou augmentée par l'exploitation minière et le colluvionnement des crassiers. Cette propriété serait difficilement compatible avec le prolifèrement de l'insecte. En général, la consti- 
tution colluvionnaire et souvent sableuse des roches mère des sols, issues des dépôts d'érosion des Andes situées en pieds de versants et s'étalant dans les étroites plaines côtières, favorise un caractère filtrant peu stable pour le développement du phylloxéra. Enfin, le recourt souvent systématique à l'irrigation ne constitue pas un terrain adéquat à cet insecte qui préfère les terrains secs.

Ces hypothèses assez déterministes elles aussi, utilisées comme arguments récurrents dans la communication du vignoble chilien, n'ont jamais été validées à 100\%, et les vignes offertes aux visiteurs lors des tours viticoles, (voir partie 3.2), sont soigneusement greffées pour que les touristes n'introduisent par inadvertance le ravageur sous leurs semelles.

En effet, Il ne faut pas négliger qu'une partie du vignoble est greffée quand même, pour prévenir, anticiper une éventuelle épidémie, pour se prémunir également des dégâts causés par les nématodes, vers parasites qui peuvent s'introduire par les racines. De manière plus organoleptique et commerciale, la vigne greffée permet d'obtenir des raisins correspondant aux standards vitivinicoles induits par la sélection post phylloxérique et ainsi mieux coller au marché local et mondial. Les vins post phylloxériques sont réputés plus accessibles, plus facilement buvables que ceux issus de vignes non greffées, simplement à cause de l'habitude de goûts qui sont arrivés après le phylloxéra et demeurent dominants aujourd'hui.

Le Chili prétend souvent posséder environ une proportion de 90\% de vignes franches de pied. La ramener aux alentours de la moitié semble un peu plus réaliste avec nos observations réalisées, y compris dans des vignes âgées de plusieurs dizaines d'années, photos 1. D'autant plus que l'Argentine contient aussi une proportion semblable à peu être plus importante, de vignes non greffées. Le Chili n'est pas seul dans cette configuration d'une relative protection du phylloxéra, L'Argentine voisine communique avec les mêmes arguments, ainsi que l'Uruguay.



Photos 1. A gauche, dans les vignes du domaine Undurraga, certains ceps poussent sans être greffés, la tige droite de la liane l'atteste. A droite, chez Concha y Toro, ces pieds Carignan sont greffés, la cicatrice de greffage se distingue dès le cep sorti de terre (Clichés E. Rouvellac, 2013). 
Cette communication visant à présenter le vignoble chilien comme sain face aux autres en quelques sortes malades s'illustre dans le détail à travers un cépage européen encore cultivé au Chili, la Carménère.

\subsection{L'originalité entretenue autour du cépage Carménère}

La Carménère est un cépage du Sud ouest de la France qui comme beaucoup de cette région possède des origines pyrénéennes basques, c'est une variété de Cabernet, figure 3, (Lavignac, 2001). Il s'épanouissaient essentiellement dans le bordelais avant le phylloxéra et classiquement, avait été emporté dans les bagages des migrants partis dans les Amériques. Il était donc arrivé au Chili avant l'épidémie de phylloxéra et tomba dans l'oubli même s'il a continué d'être cultivé, confondu avec ses géniteurs Cabernets et surtout son voisin Merlot.

Il tombe aussi dans l'oubli de l'autre côté de l'Atlantique, mais en connaissance de cause. A l'issue de la crise phylloxérique, il fait partie des variétés difficilement adaptables au greffage, possédant de plus des problèmes d'irrégularité de rendements et de coulure, liés à son caractère de mûrissement trop tardif par rapport aux standards de l'économie moderne qui se met en place petit à petit au XXème siècle. Ces problèmes sanitaires amplifiés par le climat océanique bordelais ne se posent pas de la même manière dans l'ambiance chilienne, où la vigne pousse sous un climat ressemblant plus à celui du sud de l'Espagne, très sec. Et les bons vins chiliens de Merlot, aux gouts de fruits rouges, ont longtemps caché une proportion non négligeable de Carménère.

La Carménère ne sera redécouverte au Chili que dans les années 1990, grâce aux travaux de J-M. Boursiquot. Depuis, et d'autant plus s'il est plantée non greffée, les Chiliens essaient d'en faire leur originalité et leur typicité en matière d'encépagement. De plus, le cépage participe à une communication hygiéniste et identitaire autour du vin et de la vigne au Chili.

Pourtant au départ rien n'est gagné, la Carménère, malgré un climat propice, garde des difficultés à murir et donc à être vinifiée dans de bonnes conditions. Les onologues s'accordent sur des qualités très satisfaisantes qui peuvent voir le jour une fois le vin fini, malgré des risques d'arômes végétaux parfois très présents. La superficie de Carménère stagne d'ailleurs actuellement autour de 7000 ha car elle est arrachée dans les terroirs jugés les plus tardifs.

Ce tableau peu reluisant paraît être compensé par la mise en avant de ce cépage atypique dans le marketing vitivinicole général du pays. La communication insiste sur le parcours de cette plante, oubliée par un des vieux leaders de la viticulture moderne, la France, où la Carménère ne représente que quelques ha dans des conservatoires ampélographiques, stigmatisée par la maladie phylloxérique, elle renait dans un pays du nouveau Monde et est mise en avant dans un Chili qui se veut exempt de la maladie, sain, en étant même plantée franche de pied. 
Ces aspects hygiénistes de la communication fonctionnent plutôt bien auprès du grand public et constitue une approche végétale et biologique qui s'adresse aux amateurs qui se veulent éclairés, et qui sont eux aussi sensibles à ce discours. Nous avons pu le constater en fréquentant les participants à travers d'autre biais qui font la force de la communication vitivinicole du Chili, les visites de chais à l'architecture particulière ou les tours viticoles qui organisent les visites des grands domaines chiliens, essentiellement à proximité de la capitale Santiago.

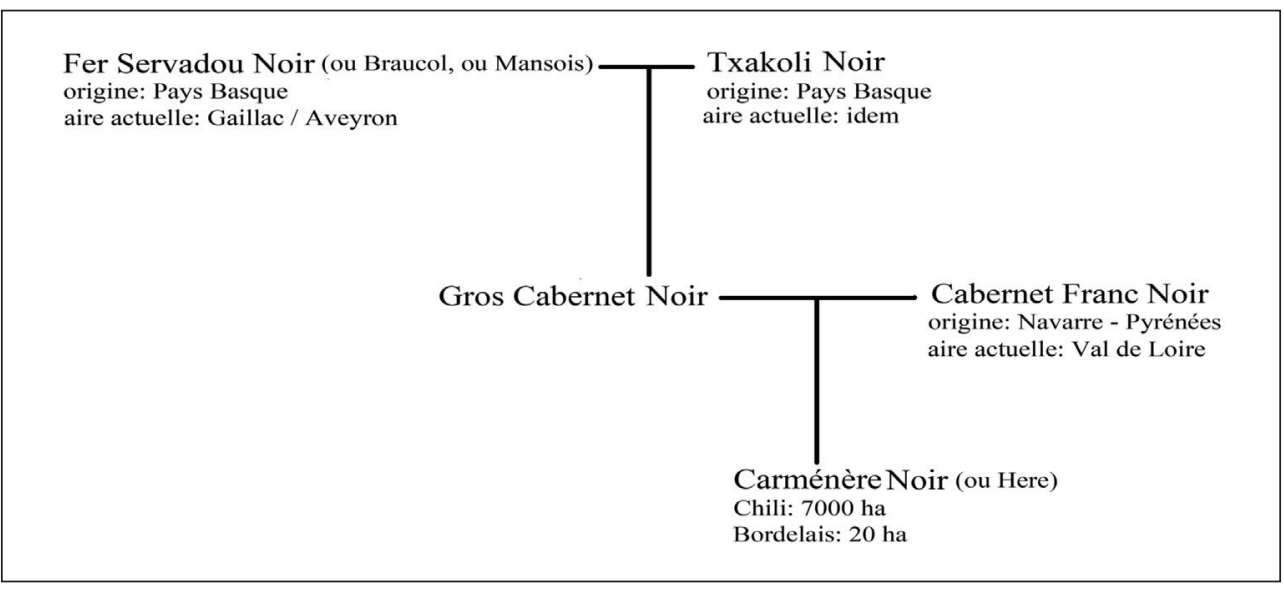

Figura 3. La généalogie du cépage Carménère.

Source: E. Rouvellac, 2015.

\section{Une communication " paravitivinicole " développée}

Comme dans beaucoup de pays du Nouveau Monde, spécifiquement aux États-Unis ou en Argentine (Schirmer, 2008, Lignon-Darmaillac, 2009, Rouvellac, 2013), l'absence de racines historiques profondes, à l'européenne, avec un patrimoine souvent multiséculaire, voire bi millénaire, est palliée par des réalisations architecturales voulant frapper le visiteur et contrastant volontairement avec le paysage ou les canons architecturaux locaux. Il en est de même dans la volonté de communiquer avec de plus en plus d'éléments extra viticoles, relativisant le poids de la vigne et du vin, et donnant un côté post moderne au markéting vitivinicole, et même au paysage.

\subsection{L'architecture iconoclaste comme entrée vitivinicole}

Le Chili ne fait pas exception au courant planétaire qui valorise la vigne et le vin à travers l'architecture des ces domaines. Dans les pays du nouveau Monde, les bâtiments 
à forte valeur ajoutée n'existaient que peu, à part peut-être en Afrique du Sud, où l'architecture traditionnelle néo coloniale domine dans les estates. C'est dans le nouveau Monde que ces premières "Cathédrales du vin " furent érigées. Au fur et à mesure de l'ouverture à la concurrence dans la deuxième partie du XXème siècle, pour remplacer les châteaux en vielles pierres inexistants et lutter contre la tradition si chère aux concurrents européens, les californiens (Opus One, Clos Pegase, Dominus Estate...) créèrent des caves d'architecture exceptionnelles avec des matériaux novateurs pour ce genre d'industrie. On peut y croiser des chais en forme de château fort médiéval (Castillo de Amorosa) ou de temple grec (Darius Winery). La palme revient peut-être aux à la bodega Catena Zapata à Mendoza, en Argentine, en forme de pyramide précolombienne.

Le Chili ne fait pas exception avec par exemple les colonnades aux pieds de ses vignes de Vina Indomita qui interpellent l'automobiliste entre Santiago et Valparaiso, (photos 2).
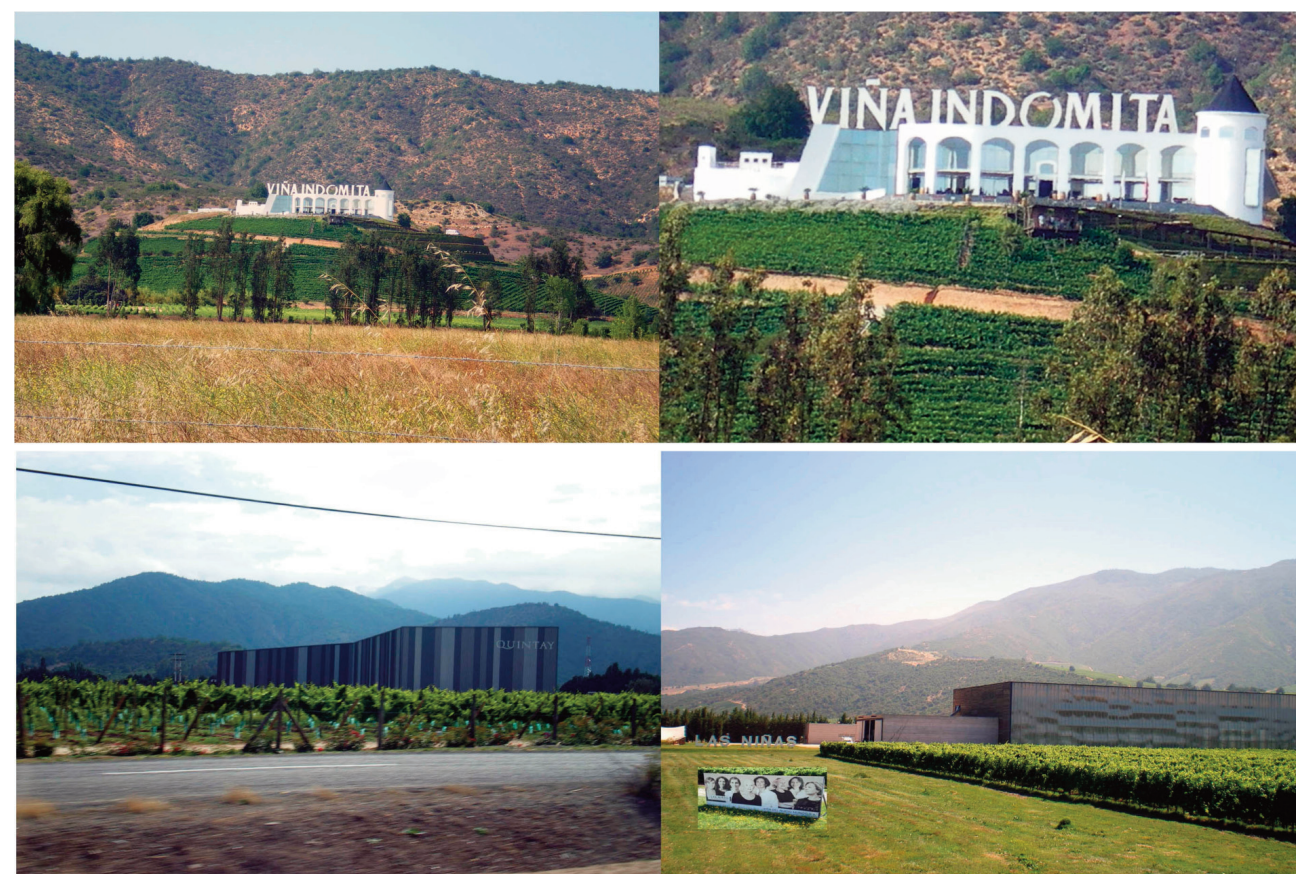

Photos 2. En haut la bodega Indomita, très ostentatoire sur la route de Santiago à Valparaiso, (DO Casablanca), en bas les bodegas Quintay (DO Casablanca) et Las Ninas (DO Colchagua) qui veulent trancher, par leur architecture cubique, sur les arrondis des montagnes pré andines à leur arrière plan. (Clichés E. Rouvellac, 2013). 
La Bodega Indomita demeure très connue au Chili pour son volume imposant, son architecture néocoloniale à colonnes, sa couleur blanche qui tranche sur le paysage, les lettres du domaine surdimensionnées, telles une publicité tapageuse d'entrée d'agglomération.

De manière moins provocatrice, L'architecture cubique est là aussi largement exploitée, voulant simplement se détacher des courbes du relief avoisinant et donnant souvent des chais en formes parallélépipédiques, comme les bodegas Quintay ou Las Ninas (photos 2).

Cette tendance " archaitecturale "2 devient planétaire et touche aujourd'hui aussi l'Europe, (Rouvellac, op. cité), que ce soit par volonté de s'intégrer dans l'environnement ou de se démarquer. D'autres jouent sur le mimétisme avec le tonneau, grâce à des bâtiments circulaires, comme Clos Apalta au Chili, ou Vinas Real dans la Rioja espagnole, ou encore la cave des Vignerons du Vallon, dans l'AOP Marcillac en Aveyron, dans le sud ouest français.

Hors des sentiers battus des bâtiments traditionnels de la maison plus ou moins bourgeoise, ces chais abritent salles de dégustation et de vente, sur lesquelles est adossé le chai de vinification, d'embouteillage et de stockage. Beaucoup de ces bâtiments se veulent voyants, produits d'appel. Cette stratégie de communication ne mise pas, ou compte peu, sur la vision classique de la vitiviniculture à travers les terroirs et les paysages comme vitrine de la production. Les chais, salle de dégustation et de vente sont associés à une mise en scène culturelle, à travers l'architecture particulière des bâtiments, mais aussi souvent à travers l'organisation d'expositions, de conférences. Ces domaines viticoles s'accompagnent de restaurant, d'hôtellerie, d'organisation de sports de pleine nature, de musée, d'œenothèque...

Oublié le territoire, le paysage ou le pays, ils sont passés au dernier plan devant le poids de la communication et du marketing dans des domaines qui peuvent y investir au point de presque faire reculer le vin au rang de prétexte, d'alibi culturel (au sens noble du terme). Ceci permet de mettre en valeur une mise en scène touristique, patrimoniale, où se mêlent la viticulture à travers des paysages, le vin à travers des chais à architecture avant-gardiste, des manifestations culturelles et/ou festives, de l'hôtellerie... Bref, nous avons presque à faire à un concept d'œnotourisme intégré, où tout est pris en compte, de la fabrication du raisin à la valorisation du vin par tous les chemins possibles de l'imagination. La communication passe même parfois par un artifice incongru qui anime la curiosité du visiteur, comme à la Bodega Santa Cruz qui organise le trajet vers la visite du domaine en téléphérique, dominant de quelques dizaines de mètres les vignes alentours.

2. Jeu de mot assumé par le site "http://archaitecture.over-blog.com" consacré aux initiatives architecturales en milieu vitivinicole. 
Visant une clientèle assez aisée, ce nouveau tourisme vigneron permet de viser des revenus supplémentaires en complément de la vente du vin et représente des arguments marketing de poids. L'œnotourisme connaît un engouement à travers la création de routes des vins, des prix d'œenotourisme ${ }^{3}$. La mise en scène du patrimoine, alliant tradition vitivinicole et architecture, activités inattendues devient un argument économique de premier ordre, (Lignon-Darmaillac, op. cité).

Il ne faut pas oublier que des bâtiments d'exploitation très classiques existent aussi et forment par exemple la vitrine de Wine Cellars en Californie, de Michel Torino en Argentine, des bodegas Undurraga ou de l'historique Concha y Toro à Santiago du Chili. Ici l'accent n'est pas mis sur un extérieur tape à l'œil, mais sur l'histoire, le patrimoine, la visite des bâtiments et de la propriété des immédiats alentours à travers un tour viticole.

\subsection{La force des tours viticoles}

C'est une forme de communication intégrée qu'offrent les tours viticoles chiliens. Essentiellement à partir de la capitale, des minibus avec leur guide font le tour des hôtels pour amener les touristes visiter de grands domaines. Le discours est d'abord axé sur l'ancienneté du domaine, relativement à la conquête espagnole, et les vertus de la lignée familiale qui a su construire et garder une histoire vitivinicole.

La vigne est souvent mise de coté, peu abordée. Concha y Toro y consacre quelques minutes au début de la visite en traversant une partie de son parc planté des principaux cépages cultivés au Chili, à la manière d'un conservatoire vitivinicole. Undurraga fait faire un crochet au bord de ses parcelles, à la fin de la visite, pour montrer la même chose, avec en plus une coupe de sol. L'accent est mis en priorité sur l'architecture coloniale, classique, des bâtiments imposants, demeures anciennes et chais des maitres des lieux. Suivent des déambulations dans les parcs arborés, jardinés qui les entourent, avant de présenter la vinification et les chais de barriques. Le public est alors amené à la dégustation et à l'achat du vin et de produits dérivés.

La vigne en tant que plante et support physique du raisin, garant de la base de la qualité du vin, est reléguée au second plan. Seuls sont rappelés les caractéristiques d'isolement et d'absence de phylloxéra, arguments récurrents de salubrité et donc de qualité dans le discours chilien.

La bodega Undurraga va jusqu'à inclure dans sa visite un arrêt devant des statues de bois représentant des dieux amérindiens mapuches, pour insister sur le lien supposé

3. Par exemple le Prix national de l'œenotourisme est de distinguer des démarches individuelles ou collectives, exemplaires, contribuant à la promotion de celui-ci. 
entre l'importance de la "Panchamama ", la déesse de la Terre dans le panthéon amérindien, et l'influence du sol dans la qualité des vins. Ce raccourci entre la culture du colonisé et celle du colonisateur, qui se veut presque syncrétique, peut même être jugé de mauvais aloi si est étudié de plus près la condition sociale et économique des amérindiens, main d'œuvre souvent taillable et corvéable à merci dans la société chilienne.

La force de cette forme d'œenotourisme est de proposer un tout en un, et qui s'aventure au delà du vin en lui donnant une épaisseur historique, en l'associant aux parcs et jardins savamment organisés, presque à la française, en mettant en avant les bâtiments imposants des propriétaires, suggérant la certaine douceur de vivre à la Scarlett Ohara dans "Autant en emporte le vent " avant la Guerre de sécession américaine, photos 3.

Tout ceci peut-il conduire à une " disneylandisation " du vitivinicole et par glissement, des territoires, des paysages ? Cette monumentalisation des bâtiments œnologiques ne connaît pas de limites surtout dans les pays du Nouveau Monde, où la fameuse Napa Valley californienne est parfois qualifiée de "parc viticole ", (Vanneph, 2012). La communication à travers l'architecture, les parcs jardinés ou la récupération des cultures locales aviticoles oublie, ou effleure seulement la vigne. Elle s'appuie sur la découverte des paysages et du patrimoine local, et aussi sur des expositions, performances artistiques, concerts, de la restauration, de l'hôtellerie, de la vente d'autres éléments que le vin, produits alimentaires locaux, bijoux, souvenirs de tous ordres.

Petit à petit, cette communication s'étoffe d'un élément indispensable dans le discours concernant toute production agroalimentaire, la prise de conscience environnementale, fut elle sincère ou seulement politiquement correcte.

\section{Vers une communication environnementale, et environnementaliste ?}

\subsection{Objectif 2020 : la charte viticole en développement durable, vraie volonté ou " greenwashing " ?}

Le Chili est le $9^{\text {ème }}$ producteur mondial de vin avec un volume de production qui s'est élevé à 10,5 millions d'hl en 2014, en baisse (-18\%) par rapport à 2013 en raison de conditions climatiques défavorables. Le pays était également en 2014 le $4^{\mathrm{ème}}$ exportateur mondial de vin en volume avec 8 millions d'hl, (OIV, 2015), juste derrière la France avec 14 millions d'hl. La viticulture est l'un des atouts du Chili à l'export et ses exportations de vin augmentent régulièrement, en particulier vers l'Europe et l'Asie. Le pays importe peu de vin, le marché étant saturé par la production locale. En charge de promouvoir l'image des vins chiliens dans le monde, l'association "Vins du Chili " a établi un plan stratégique pour renforcer la compétitivité du secteur vinicole chilien et consolider sa place d'industrie durable et de qualité d'ici 2020. 

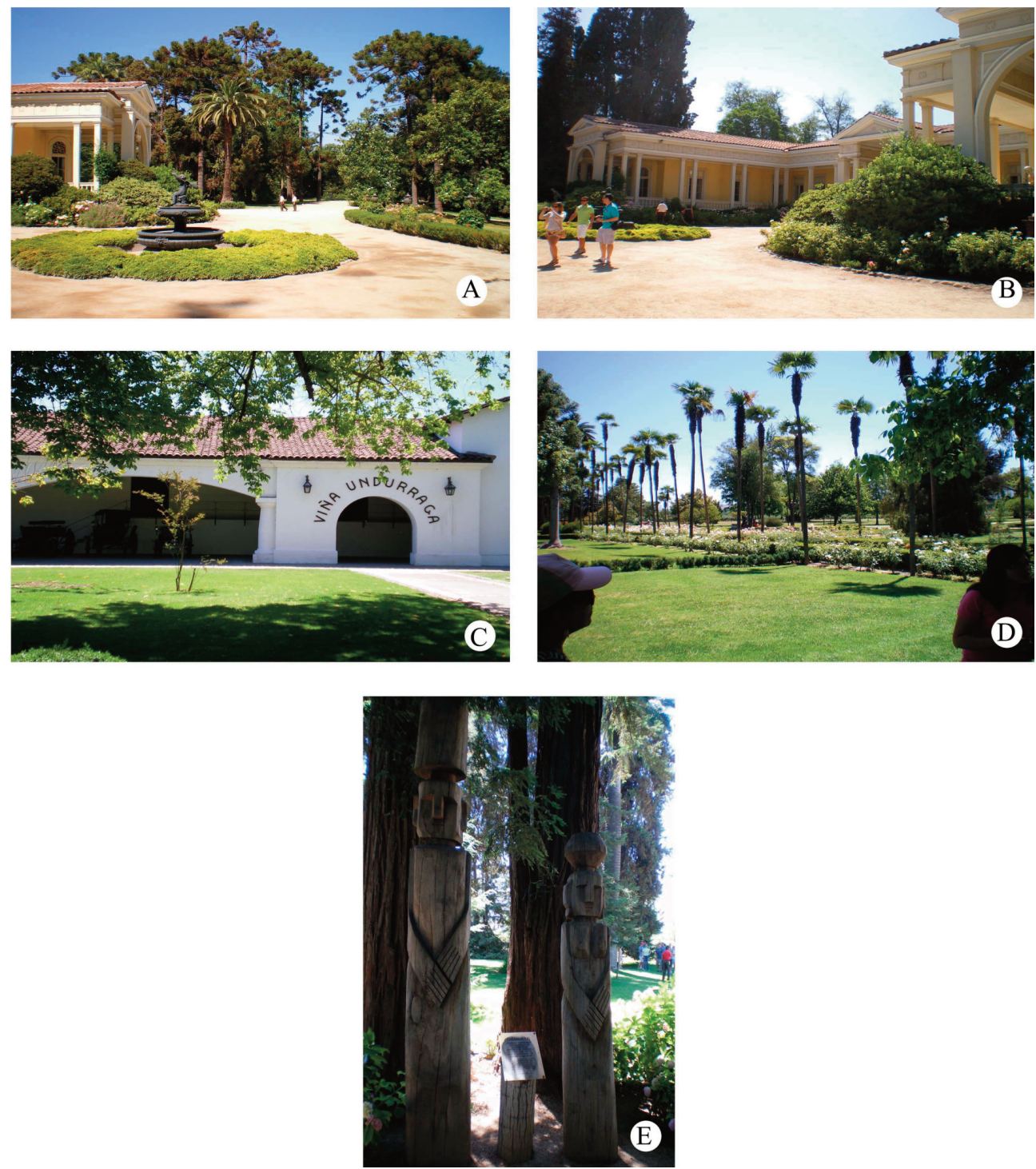

Photos 3. Les bodegas Concha y Toro (A et B) et Undurraga ( $C, D$ et $E$ ) mettent I'accent lors du tour viticole de leur domaine sur l'architecture classique, néocoloniale, de leurs bâtiments d'apparat. Le classique et I'historique sont mis en avant par exemple par l'alignement de calèches qui se distingue sous le porche d'Undurraga (C). Chaque fois est mis en scène une promenade dans le parc arboré, gage d'un luxe certain auquel est associé le vin, présent uniquement en fin de parcours. Son même convoqués les divinités mapuches (déesse de la terre), (C), pour assurer un lien imaginaire entre l'environnment amérindien et le vin européen. (Clichés E. Rouvellac, 2013). 


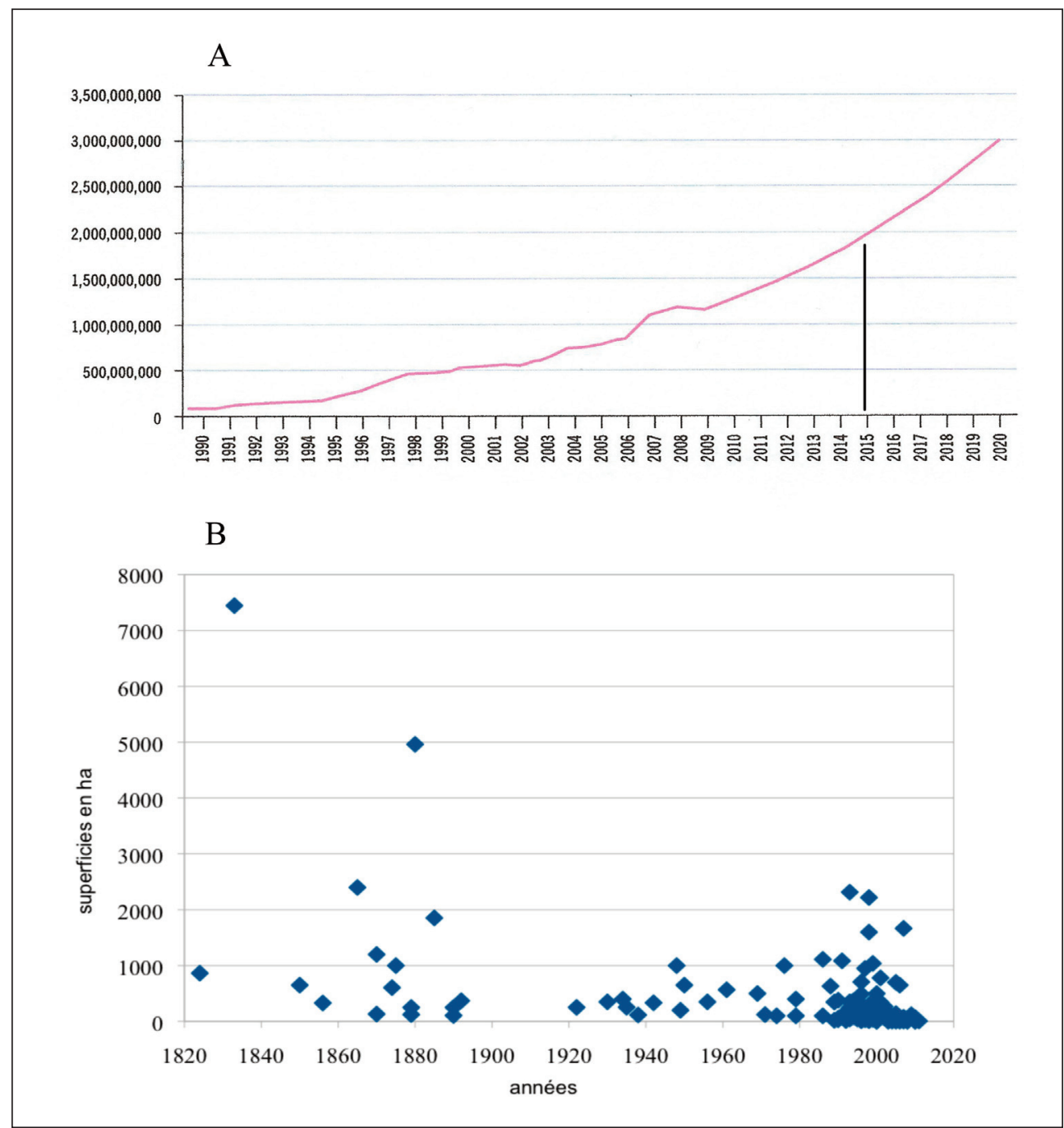

Figura 4. A : Evolution historique et projection du nombre de bouteilles de vins exportées au Chili.

B : Croisement des superficies et des années d'etablissement de 143 bodegas chiliennes. Source: A: Wines of Chile, 2013. B: E. Rouvellac, 2015.

Depuis 2011, l'industrie vitivinicole chilienne souhaite devenir une référence en atteignant le premier rang mondial d'exportateur de produits vitivinicoles labellisé développement durable (figure 4). Pour cela, une charte octroyant la certification officielle : " développement durable chilien " a pour but de mettre en place progressivement des pratiques durables. Cette charte a été rédigée par l'organisation Vinos de Chile, qui re- 
groupe 92 entreprises représentant 90\% de la production vinicole nationale. Ses dispositions se veulent à la fois écologiques, sociales et économiques à travers les trois volets de la Charte : le vignoble, la cave et les responsabilités sociales. D'ici 2020, elle espère réunir 3 milliards de dollars d'exportations de bouteilles de vin. Le plan élaboré prévoit pour y arriver un taux de croissance annuel de 9,2\% qui sera basé sur le renforcement de la reconnaissance et de l'image des vins du Chili comme une appellation de niveau mondial. Cet objectif s'organise autour d'un plan en 4 axes:

- Les atouts naturels du territoire.

Une diversité du territoire et une qualité accrue des produits qui prétendent s'appuyer sur les avantages agroclimatiques du Chili. Etirés sur près de 1200 km de latitude, Les terroirs viticoles tirent leur réputation du grand contraste paysager assuré par la proximité de l'océan et de la Cordillère. Ils bénéficient d'une diversité géomorphologiques, pédologiques, climatiques qui peut servir à produire une large variété de vins. Les progrès mondialisés, sans cesse croissant dans le domaine de la qualité du vin, bénéficient aussi au Chili.

- Le développement durable mis sur le devant de la scène.

L'industrie du vin chilien a pris conscience de la protection de l'environnement et veut intégrer dans toutes les étapes du cycle de production, de la vigne au verre, les pratiques du développement durable. Un programme a été proposé pour fournir un cadre commun afin d'orienter la viticulture vers des pratiques écologiques visant à l'agriculture biologique. La vinification est axée vers plus d'efficacité énergétique, vers la conservation des ressources, sur le souci d'alléger le bilan carbone de la production et de gérer les déchets. Ce programme de développement durable est certifié par un code national le "WOC certified sustainable ", apposé sur les bouteilles des producteurs respectant cette charte.

- L'œnotourisme comme atout maitre du marketing.

La diversité géographique, l'exotisme du vignoble chilien joue un rôle important dans le tourisme. Le plan d'élaboration de la charte 2020 prévoit également dans son programme d'accroitre l'œenotourisme. L'objectif est de développer la réputation des vins chiliens tout en profitant des territoires, de l'aménagement des paysages viticoles en associant des images et des vins. Et comme nous venons de le voir, le Chili développe depuis de nombreuses années maintenant des tours viticoles, surtout à travers des grands domaines, pour promouvoir sa réputation vitivinicoles.

- L’innovation toujours encouragée.

L'industrie chilienne, pour contribuer au succès de ses vins, veut investir dans l'innovation et la technologie pour améliorer le processus et les normes de production de qualité. Afin de poursuivre cette avancée, ont été créés Vinnova et Tecnovid technical 
consortiums pour développer des projets de recherche et développement qui répondent aux besoins des vignobles en les mettant en relation avec les chercheurs. La première phase de ce projet couvrait les domaines de compétence tels que la viticulture, l'œenologie, l'environnement et le marketing. La deuxième phase analyse actuellement les ressources génétiques, le développement durable, le terroir, les préférences des consommateurs, (Vitisphere.com, 2012).

\subsection{Des résultats en demi-teinte, où la communication environnementale sert l'exportation}

L'organisation "Wine of Chile " a ressenti récemment la nécessité de créer son code de durabilité et son sceau, dans le but de renforcer son image et de gagner en compétitivité, plus particulièrement aux Etats-Unis, au Royaume-Uni et dans les pays nordiques. Un code général comprenant 17 points critiques a été créé, reprenant un ensemble de 119 critères au total, Les organismes producteurs qui satisfont au moins 60\% de ces critères sont certifiés. Ce code est divisé en 3 signalétiques :

- Le vert pour les pratiques culturales axées sur les ressources naturelles et l'économie d'énergies, avec l'économie de matières premières, le calcul d'un ratio entre matières premières utilisées et matières premières recyclables, avec un meilleure maitrise de l'irrigation, du traitement des eaux usées, avec une quantification des traitements, une meilleure protection des sols et de la biodiversité.

- Le rouge pour les pratiques œnologiques axées sur l'efficacité énergétique, la gestion de l'eau et le recyclage des déchets.

- L'orange pour les pratiques sociales axées sur l'éthique des conditions de travail. C'est le volet le plus rempli qui essaie de balayer tout l'éventail, à travers un meilleur encadrement des types de contrat de travail, la mise en place d'aides sociales de tous ordres, prendre en compte les syndicats, la représentation des salariés et le dialogue social, quantifier les négociations salariales, rationaliser et limiter les écarts de salaire, favoriser la formation, la place des femmes, la liberté d'association, lutter contre la discrimination, le travail des enfants, le travail forcé, relancer la lutte contre les accidents du travail et quantifier leur coûts, se préoccuper davantage de la santé des salariés.

Ces injonctions suivent et citent dans leur ensemble les recommandations de l'Organisation Internationale du Travail (OIT), et ne sont pas quantifiées. Il importe aux entrepreneurs de mettre en avant de quelle manière et à quelle hauteur ils les ont mises en place.

Malgré son ambition affichée, la certification durable chilienne reste encore imparfaite. En 2013, 14 entreprises pouvaient afficher le label "vin chilien certifié durable ", 
mais ne répondaient en fait qu'au premier volet d'exigences de la charte, celui concernant les seules pratiques viticoles. Les autres parties (travaux de chais et responsabilités sociales) devaient être certifiées au cours de l'année. 24 autres domaines étaient aux prémices de similaires démarches de certification. L'obtention à plus long terme de cette certification restera cependant incomplète. En effet, certains vignobles pouvant être certifiés alors que 60\% seulement de leurs surfaces répondent à la démarche durable. Les premiers vins certifiés durables le sont-ils réellement?

Cette charte affiche aussi clairement une volonté de rompre avec des pratiques de gestion de la main d'œuvre viticole tendant à exploiter et à ne pas respecter les personnes. Sont concernées en premier lieu les amérindiens surtout Mapuches qui constituent une main d'œuvre sous payée, sous considérée dans le pays.

En effet, les vins chiliens bénéficient d'un rapport qualité/prix favorable. Ils se sont ainsi imposés auprès d'une large palette de pays consommateurs. Les coûts de production au Chili sont peu élevés, de 1430 à $3200 € /$ ha contre 5000 à $7000 € /$ ha dans les vignobles du Sud de la France par exemple, (IFV Midi-Pyrénées, 2013). Le poste principal de charge est la main d'œuvre qui représente 50\% des coûts. Le revenu d'un ouvrier est de 20 \$ par jour à raison d'un ouvrier permanent pour 10 ha de vigne. Ceci représente des coûts de production par $\mathrm{kg}$ de $0,2 \$ / \mathrm{kg}$ de raisin en viticulture traditionnelle. Cela peut expliquer pourquoi la mécanisation est encore peu répandue au Chili malgré l'avancée de l'emploi de la machine à vendanger. Les prix pour le consommateur français oscillent entre 4 et $8 €$ la bouteille pour des vins de moyennes gammes, ce qui les rend très compétitifs.

Même si presque 40 entreprises vitivinicoles approchent les désidérata de cette charte, et ont émis l'ambition de la respecter, les résultats peuvent être lus soit de manière optimiste, soit le contraire, les récoltes de données montrant que les domaines concernés pour l'instant sont à la croisée des chemins. Ainsi 56\% des critères économiques de la charte sont respectés, $52 \%$ de ceux retraçant les conditions de travail et les droits humains, 56\% de ceux s'attachant à la qualité de la vigne et du vin, 40\% des critères liés à l'environnement, (Vinos de Chile, 2013).

\section{Conclusion}

Avec seulement une consommation de 171 de vin par habitant pour une population de 18 millions (contre 441 par habitant en France par exemple), le Chili, comme presque tous les pays du Nouveau monde viticole, ne possède ni un marché intérieur suffisant, ni une population assez nombreuse, pour absorber une partie de sa production qui permettrait de moins s'attarder sur ses exportations vinicoles. 
Exporter ou presque en finir avec la viticulture, tel est le défi du Chili. Les politiques dynamiques de communication et de marketing tirent leur essence de ce contexte particulier.

Emboiter le pas du développement durable pour accroitre les exportations paraît une louable intention mais les enjeux économiques de la filière permettent de douter de la complète sincérité de la démarche. Il reste à savoir si une part assez importante de la clientèle étrangère devient sensible à ce discours, notamment avec les vins d'entrée de gamme qui touchent plus un public moins averti. La communication intérieure utilise et instrumentiste avec brio les conditions naturelles, en restant à une échelle suffisamment petite pour demeurer floue, générale et dans l'indémontrable. Le classicisme de la communication des tours viticoles assure une vision finalement très européenne, (les Mapuches en plus), très axée sur l'antériorité, à destination d'une clientèle éclairée et/ou à pouvoir d'achat non négligeable.

Quoi qu'il en soit, le Chili viticole démontre ainsi qu'il appartient définitivement au monde viticole contemporain, il en est même, par les pans d'originalités qu'il cultive, un élément innovant et moteur, pouvant potentiellement donner des leçons à l'ancienne Europe.

L'évolution récente repose sur des locomotives historiques et rappelant encore les latifundia. Mais la création de nombreux nouveaux domaines, (figure 4), dans un laps de temps ramassé depuis la fin de la dictature militaire (1990), qui sont essentiellement des petites structures, petites bodegas, voire des vignerons s'inspirant du mouvement " garagistes ", montrent le dynamisme et les opportunités commerciales et socio économiques que peut offrir le secteur vitivinicole chilien.

\section{Bibliographie}

Hinnewinkel, J.C. et Velasco-Graciet, H., 2007. Espace et temporalités du vignoble: une comparaison franco-chilienne. Géoconfluence, dossier : "Le vin, entre sociétés, marchés et territoires ". http://geoconfluences.ens-lyon.fr/doc/typespace/vin/VinScient11.htm

Hinnewinkel, J.C. et Velasco-Graciet, H., 2005. Les vins de l'hémisphère sud et la mondialisation de la planète vitivinicole. Cahiers d'Outre-Mer, Vol. 58, n 231-232, Presses Universitaires de Bordeaux, pp. 267-280, Bordeaux.

Lavignac, G., 2001. Cépages du Sud-Ouest, 2000 ans d'histoire. Edition du Rouergue et Edition INRA, 272 p. Rodez.

Lignon-Darmaillac, S., 2009. L'œenotourisme en France, nouvelle valorisation des vignobles, Edition Féret, 255 p. Bordeaux.

Organisation Internationale de la Vigne et du Vin (OIV), 2015. Éléments de conjecture mondiale 2014. http://www.oiv.int/public/medias/2934/oiv-noteconjavril2015-fr.pdf 
Paegelow, M. et Toro-Balboutin, D.E., 2008. Essor et restructuration du vignoble chilien: l'exemple du Maule (VIIème région) 1995-2005. Cahiers d'Outre-Mer, Vol. 58, n 231-232, Presses Universitaires de Bordeaux, pp. 301-328, Bordeaux.

Rouvellac, E., 2013. La dissolution des terroirs et des territoires viticoles dans l'architecture des domaines viticoles. Sud-Ouest Européen, n 36, Presses Universitaires du Midi, pp. 85-96, Toulouse.

Salomon, J.N., 2005. Nouveaux vignoble et évolution des anciens face à la mondialisation. Cahiers d'Outre-Mer, Vol. 58, n 231-232, Presses Universitaires de Bordeaux, pp. 397-418, Bordeaux.

Schirmer, R., 2005. Le Chili, un vignoble à la conquête du monde. Cabiers d'Outre-Mer, Vol. 58, n 231-232, Presses Universitaires de Bordeaux, pp. 301-328, Bordeaux.

Schirmer, R., 2008. Châteaux bordelais et vina chilienne : la convergence des modèles. Historiens et géographes $\mathrm{n}^{\circ}$ 402, pp. 193-204, Paris.

Tulet, J.C. et Sanchez Alvarez, J., 2002. La croissance du vignoble du Chili par l'amélioration de la qualité et de l'exportation. Sud-Ouest Européen, n 14, Presses Universitaires du Midi, pp. 11-21, Toulouse.

Vanneph, A., 2012. In: "Tourisme du vin ". Ecologik, n² 27, Edition Avivre, pp. 63-92, Paris.

Vinos De Chile, 2013. La industria del Vino y los Reportes de Sostenibilidad. http://www.winesofchile.org

Vitisphere.com, 2012. http://www.vitisphere.com/actualite-77769-Chili-le-marche-du-vin.htm 